\title{
LA HOUILLE BLANCHE
}

\section{Revue générale des Forces Hydro-Electriques et de leurs applications}

6e Année. - Juin 1907. - No.6.

La Houllle noire a fait l'Industrie moderne; la Houille blanche la transformera.

\section{A propos de l'Aménagement des Montagnes}

Giscours. prononcés an Sénat, le 18 janviér $1907, \mathrm{par}^{\mathrm{N}} \mathrm{MM}$. Aublwkned et Flaissieres, sénateurs, Métine, ancien ministre, el RuAu, Ministre de I'Agriculture.

\section{DISCOURS DE. M. MÉLINE}

Messieurs, je commence par remerciel" la commission des fnances d'avoir bien voulu, hier, se preter au renvoi d'une question qui touche à des pablèmes de l'ordre le plus important ef qui est digne de retenir un instant l'attention du Sénat. Ces problèmes passionnent de plus en plus l'opinion, réveillée chaque année par des catastrophes, des sinistres dont l'intensilé croissanle prouve manilestement l'insulflsance des moyens employés à les conjurer".

Cette année a été particulièrement douloureuse. Après une sécheresse persistante, qui a causé lant de pertes à nos agriculteurs, des pluies torrentielles se sont précipitées du baut des montagnes dans les vallées, sans rencontrer de résis" lance, provoquan partout des inondations, des éboulements, des glissements de terrains, qui ont ravagé les plus belles parties de la France.

Cies tristes événements ont en un écho à la Chambrest des députés, el les représentants des régions intéressées ont proposé un relevement de crédit de 175.000 fr". que la commission de la Chambre des députés avait acceplé.

Devant la. Chanbre, M. le ministre des finances a opposé au vole de ce crédit une résislance lellement irréductible que les auteur's de l'amendement ont dû reculer. 1ls se sont contentés du vole d'une angmentation de crédil de $1.000 \mathrm{fr}$., indiquant la volonté de la Chambré des députés do voir le Gouvernement lui apporter l'année prochaine une proposition lerme.

Votre commission des finances a supprimés ce crédil de $1.000 \mathrm{fr}$., parce qu'ji est del jurispindence, au Sénat, qu'aucun crédil d'indication ne pent etre infroduil dans le budget. Mais, toul cn repoussant le crédit, lhonorwble rapporteur du budget de l'agriculture, M. Jean Dupay, a laissé entendre qu'il n'etait nullement hostile à une proposition de relèvement pour l'avenir. Il suffit de savoir quelle altitude M. Jean Dupuy a prise dans ces questions, quand il élait ministre de l'agriculture, pour" considérer l'espéranco qu'il nous donne comme un engagement pour l'année prochaine.

Mais, Messieurs, je liens à dire tout de suile qu'alors même que nous obtiendrions l'année prochaine, ce qui sans doute ne sera pas facile, le r'elèvement de crédit qu'on peut prévoix, la question ne serait nullement tranchée, car, à mon avis, le problème h'est pas là. Il est moins dans l'importance du. crédit que dans l'emploi qui en est fait.

Vous n'ignorez pas, en effet, que l'emplot du crédib inscrit à ce chapitre de la conservation ef de la restauratilon des montagnes est réglé par une loi de 1882. Cette loi est une loi spéciale, qui s'applique à des travaux d'une naluré particulières. Enlle a pour but de venir au secours des montagnes qui tombent en ruines, qui n'ont plus de végélation d'aucune sorte, qui menacent de glisser dans la plaine, et dont les flancs se sont transformés en torrentis dévaslateur's ; aussi les opérations entreptises avec ce crédit ont-elles en généra! pour objet de corriger, d'éteindre les torrents, selon la formule consacrée. Je ne songe pas à nier l'ulilité de grands travaux of je me plais à reconnaîu"e que l'administration des forêts a élé pour leur exéculion à la hauteur de sa tâche.

Ses agents on prouvé qu'ils avaiont toutes les compétences et qu'ils élaient, au besoin, des ingénieur's de premier ordre. Mais ce qu'il faub bien que je signale a l'allenlion du Sénal, c'est que l'application de celle loi est forcoment restreinle à des cas lines pelu nombreux ot très limilés. Les grands torrenls quo la loi de 1882 a en vae n'existent quo dans quelques rures département, of dans cesi départements eux-mêmes, ils sonl à l'étali d'exception.

Les travaux qui sy appliquent sond d'aulant plus limiles que la dépense qu'ils engagent est extremoment considérable ; l'extinction de certains do ces torrents a coulé jusqu'à 1 million. Vous devinez combien lachion de l'administration est bornéc, si vous rapprochez celte dépense de 1 million du crédil lui-mème qui n'est que de 3 millions par an.

En résumé la loi do 1882 n'onère guère que dans une di. zaine de départements el sur des poinls très limilés de ces départements.

El maintenant, si nous délournons un inslant nos regards de ces départements, si nous les promenons sur" l'cn. semble du pays, que voyons-nous? Nous volyons non pas quelques montagnes, mais des milliers de monlagnes dénudées, ravinées, où la lorre esl remplacée part la roche nue ou la lande, où l'eau n'ost relenuo par rien, el glisse jusqu'au lond dos vallées sans que rien l'arrote; il en résulte que, par une élrange contradiction, les sources se tarissent, on mème temps que l'inondation detruil tout sur son passage.

C'ast que ces momlagnes ont perdu ce qui faisail leur vérilable protoction, l'herbe, le gazon, les pâlurages ; elles ond perdu ce feutre épajs et ondoyanh qui rehenait l'eau comme une éponge, qui la conservait et qui alimentait les sources lout en empechanl les pluies torrentielles de descendre en masse violente au fond des vallées.

Voilà le rôlo quo jouatend dans les monlagnes les gazonnements el les palurages florissants d'aubrefois. On los a négligés, on a cessé de les enlrelenir, de las culliver ; dans d'aubres régions, on en a lellement abusé qu'on les a ruinés et mis hor's d'usage.

Tel est le mal profond qui afnige les trois quarls des departements de France, je parle bien entendu des départements de montagne. Cet état de choses a eu, au point de vue agricole, les conséquences les plus déplorables. Il est pour beaucoup dans l'exode des campagnes : nos montagnards, se voyant cernés de lous côtés, par la forêt, par la lande ou par la roche, ont été ainsi poussés par une tendance invincible à descendre dans la plaine.

Autrefois, quand ils trouvaient à côté d'eux de gras palurages, ils 'y' restatient volontier's parce que sur ces gras patu- 
rages s'installaient, l'industrie beurrière, l'industrio fromagere, los fruitieres, tout un élément de prospérité qui leur assu rale un large bien-être.

Aujourdhui le mal s'élend et si on n'y prend garde, cos montagnes, qui sont encore dans ce quo j'appellerail la première partie de la maladie, ne larderont pas à arriver au second étak, l'etal désastreux, prévu par la loi de 188\%. Les torrents s'y traceront insensiblement un lit et il viendra un jour où, quand on voudra porter un remede au mal, il sera trop tard, parce que l'operations sera trop coúleuse. Si l'extinclion de quelques torrents coutc 3 millions par an, je vous lalisse à penser ce qu'il faudra dépenser lorsque des milliers de torrents se déchaineront sur toute la surface du lerritoire.

J'en ai dit assez pour laisser entrevoir le romède au mal. Le remède est dans la reconstitution des pâturages des hautes montagnes. Il faul faire uno opétation densemble sur toute la surface du pays pour rékablir, par le gazonnement, les pâhuragas de nos mon lagnes ; c'est le senl moyen d'assuror l'approvisionnement de nos sources, de régulariser le débit de nos cours des eaux, el te retenir les habitants do nos monlagnes sur les hauteurs où ils vivaient jusqu'à présent heureux ol prospèresi.

Les avantages de lopéralion sont tellement considérables qu'on a peirte à comprendra que les communes qui sont les premieres intéressés dans la queslion ne l'aient prise en main.

11 parail étrange, en eflat, que les communes négligen! des truvaux qui sont dans l'intélw non seulement de chaque agricullenr, mais encore de la collectivité lout entière.

C'osl, dilon, parce que les municipalités se désintéressen $b$ trop des questions d'intéró général, parce qu'ellos sont anx prises avec des rivalilés locales qui paralysent leur" bome volonté.

11 pout y avoir du viai dans cos considérations ; mais il faut bien reconnailre aussé que les consoils municipaux ne possèdent nullomenl les éléments nécessiares pour conduire à bien unc paroille ontrepriso. Ils n'ont mas d'abord les conmaissances lechniques suflsanks ; ils nono pas non plus sous la main les agents d'exéculion qui leur sonb indispensables. Ils n'ont pas toujours non plus les ressources nécessaires pour commoncer les lravaux ot, quand ils sont exécutess, pout les entretenir.

Il palail bien ainsi domontré que les, communes touter scules, liviées à ellos-mêmes, ne peuvent pas réaliser un progrès de oe ganre. C'est de celte constatation de fail, messieurs, qu'est sortie en 1897 l'or"ganication du service pasloral au ministère de l'agriculture. Il a élé confé à l'administrabion des forèts, qui elait toul indiquce pour" le recevoir", parce que les agents des forêls vivent dans les montagnes, à cóté des populations, qu'ils sont en relations constantes avec olles ; que, de plus, ils possedent aujourd'hui des connaissances agricoles trè̉es élendues.

Gest done l'administration des forth qui devait recevoir ce service plutòt qu'une aubre. Quel éait le ròle de ce service dans la ponsée do ceux qui lont crée ? Il était multiple. Liadministralion des forêts devail, d'abord, s'owcuper de faire l'éducalion des populations.

Elle devait préparer leur espril aux travaux qu'on allait leurn demander ; il fallait les convertir en un. mot à l'idée nouvelle., On objecte, il est vrai, que souvent les communez sont très diffciles à convaincre, qu'elles ne se résignent pas aisemont à la direction quon essaie de leur donner, et qu'elles ont témoigné d'un esprit tellement réfractaire que Fudministration a été souvent obligée de reculer.

Je conviens volontiers de ces diffcultés. Il ne faudrait cependant pas les exagererer. ot, surtout, il ne faudrait pas en tirer des conséquences pessimistes et trop décourageantes. Il faul prendre nos agricilteurs comme ils sont faits. 1ls ie sont nullement les ennemis du progrès ; seulement ils ont une cortaine déflance des choses nouvelles, et il ne les ap- pliruent que Lorsqu'on leur a bien prouvé par des résultats pratiques et indiscutables, qu'elles répondent à leurs vérilables intérèts.

Mais le jour ou ils sont enfin convaincus, parce qu'ils ont touché du doigt les avantages de la solution qu'on leur reconmande ils sont les premiers à marcher de l'avant. Ils nous ont donnć de cet entrain au progrès des preuves innombrables depuis dix ans.

Le rôle de l'administration est tout de persuasion ; il sera d'autant plus efficace qu'elle y ajoulera des propositions de nature à altir"er" les communes de son colé ; il faut d'abord qu'dla lacilibe leur tâche en leur" soumeltant des plans de travaux of qu'elle en dirige l'exécution. Si les conseils municipaux ne veulent pas entrer dans les vues de ladministration, celle-ci peut constituer des syndicals d'iniliatives composés d'labitan ls de la commune, qui foront l'opération pour le comple de la commune. Fn oulta, l'administration a à sa disposition un moven de séduction tout puissant dians les subventions qu'elle peut donner, pour les déterminer, aux communes les plus hésitantes.

Enftu, messieurs, l'administration a un autre ròle à jouer qui se rattache à une question extrèmemenl importante : celle du ruboisement, dont je vous demande la permission de dire un mol.

Jo ne voudrais pas m’étenure sur ce sujet ; je n'ai pensionne à convainore ici de la nécessité du reboisement, tout le monde déphore que cettre vaste opération, si utile au pays tout gnlier, se fasse aussi lentement, el qu'il soil si difficile d'abtenir des communes leur concours pour une cuvre qui est toute dans leur in térêt. Lá encore il faul compler avec la menlalite des populations ruiales. Il est certain qu'elles ne consentent pas volontiers à abandonner leurs palturages, meme quand ils ne valent rien ou presque rien. Si mince que soit lo protit quelles en tirent, elles veulent les garder.

Mars, messieurs, il me semble qu'il serajt facile de modificr leur point de vue si l'alministution venait leur" tenir" le langage suivant : "Vous ètes proprjétaire de vasies terrains incultos yui se composent de mauvaises ler"res, de roches mues el do landes, de terres médiones et do bonnes ferres. Or", voici le marché que nous vous proposons de faire : vous allez nous livror vos mauvaises terres, vos roches nues, nous les I"eboiscrons pour volre compte, al nous vous livrerons un jour une forèt en bon état, qui serkt une fortune pour volre commune, et qui ne vous aura rien coulé ; quand à vos bonnes terresi da à vos terres moyennes, nous allons les remeltre en bon état, les cultiver pour" vous, y créer des pâlitrages plantureux, et vous obtiendrez de ces terr'es que nous vous laissons plus de fourrag'€ que vous n'en l.touviez auparavant sur" le pâlurage tout entier" ».

Je ne peux pas croire que les conseils municipaux jess tent insensibles à de pareilles raisons; elles sont à la portée du derniter des agriculteurs.

Si on leur tenait ce langagc, el surtoul si on le leur tenait avec donceur, avec porsévérance, on aurait quelques chances d'être écouté.

C'est ainsi que se ficnl étroitement la question de lamélioration des paturages ef celle du reboisement.

Voyons maintemant ce qu'a fail ce service personnel, et ce qu'il a obtenu. Il a été installé, dans les meilleures conditjons possibles, au ministère che l'agriculture. On a mis à sa lète un agent supérieur dont persomne ne songrera à conteslier: l'activité et l'intelligence. A côté de lui, ont été placés đes collaborkteur's, qui n'étaicnl pas très nombreux mais qui etaient de premier ordre et parmi eux un forestier qui a athche son nom à la question des améliorations pastorales, M. l'inspecteur Cardot, qui a exéculé de magnifiques travaux dans le Doubs, et dans beakcoup d'autres departements.

Pour orienter la marche du service nouveau, une grande commission extraparlementaire a été constibué dont fai- 
saient partie nos honorables collègues César Duval et Antoine Perrier, quj sont des mailres en la matière et gràce à leurs lumièros al a celles de tous les hommes competonts quil faisaient nartie de cetlé commission, on a tracé au ser"vice des amélioralions pastorales un plan d'ensemble par faitement conçu, of qu'il n'y avait qu'à oxécuter avec suile et vigueur.

Voyons maintenant comment cel plan a été exécuté of ce qui a été fait.

Le décret cui a institué le service date de 1897. Les débuls: on ont été for"cément lente, laborieux et difficiles. Il a fallu passer par une période de tatonnement, qui heureusement n'a pas été très longue, car presque tout der suite le service s'est developpé. Jo le prouve par deux chiffres : en: 1897, le service pastoral navait mis à l'étude que quatro projets ; il y a deux ans, c'est plus dè doux cents projets qui ont été pirépiarés.

On a donc fail beaucoup de chemin en peu de temps, el il est inconstestable que le service a déjà oblenu des résultats yositils. Je dois dire, dailleurs, qu'il a élé encouragé ef soutonu par lous les ministres qui se sont succédé au ministère de l'agriculture, aussi bien que par le Parlement.M.Jean Dupuy cuand il étail ministre de l'agricullure, lui a témoigné une sollicitude parliculière ; quand à l'honorable ministre actuel, déjà, en 1904, dans son rapport sur" le budget de l'agriculture, il en avait signalé l'importance et, depuis qu'il est ail pouvoir, il a fait mieux encore : il l'a développé autant qu'il a pu en faisant en sa laveur des virements de crédil parlaitement réguliers, mais qui n'en élaient nas moins une hardiesse. Sans lui, en effet, le service serait resté stagnant.

A côté de ce qu'a fail l'administration par" elle-même, il faut faire entrer en ligne de compte un grand nombre de travaux entrepris par l'injtiative privée sur" plusieurs points de la France. Partout, l'impulsion donnée a produit ur. réveil vérlable. C'est ainsi que dans les Pyrénées, un véritable apôtre de l'idée pastorale, un homme aussi modeste qu'énorgique, dont le nom mérile d'êtro connu, M. Cyrille Meunier, est parvenu, par la recionstitution dos paturages et la création de fruitières, à constituer de toutes piecos une industrie fromagère qui fait la fortune de la région. Lihonorable M. Lintilhac, nous faisail, dans une de nos dernières séances, la peinture saisisante el très porétique de l'œuvre accomplie dans le Cantal.

M. Lintuhac. - Il $\mathrm{y}$ a une résistance instinctive, opiniâtre, de la part du paysan, à renoncer à la vaine pâtul"e pour accepter le reboisement, même dọ landes à peu près stériles. Lui offre-ton l'achal de ces landos, comme nous l'avons fail dans le Cantal, à 100 fr. l'hectare ? Il refuse, croyant à une arrière-pensée de lacheleur, comme si celui-ci allait découvrir un trésor dans ladite lankle et l'en fruster Jui.

Le trésor y est biten, mais à la surface $t$ là où le tenancier ne sail pas le faire surgir.

Donc il faut, comme vous le demandez, lui compenser cetle perte imaginaire par l'appât sensible d'un pâturage créé à colé dans les lerres médiocres.

Quant à l'éponge qu'est le gazon sur les hauledry, à la barrière végélale qu'est la forêt à mi-cóte, si supérieures l'une et l'autre aux couteux endiguements des ingénieurs, il n'a pas la foi, quoiqu'il ail lignorance. Il faut lui ôter cellerei pour lui donner celle-là.

M. Móline - Je suis absolument de lavis de M. Lintilhac. Si l'on rencontre quelcuefois des résistances dans les populabions, on pourrait certainement en avoir raison plus d'uno fois avec certaines précaulions. Ainsi, je sais, par exemple, qu'une des causes qui éveillent le plus les défiances des communes, c'est l'engagement qu'on leur' demande, avant d' $\epsilon$ cécuter les travaux de pâturage, de se soumeltre au régime forestier.

Les populations ne peuvent pas se résigner à se mettre sous le joug de l'administralion pour quelques bouquets do bois qu'on ajoute à leurs paturages. Il y aurait certainement un adoucissement à apporter à cette clause par lrop rigourcuse qui fait trop souvent obstacle au progrès pastoral.

Je ne peux pas parler des travaux dus à l'initialive individuelle, après avour cilé M. Cyrille Meunior, sans rendre juslice aussi à un de nos collègues, qui a fail plus que personne pour les améliorations pastorales, M. Calvet, ancien inspecteur des forets.

J'en ai fni avec la. revue des travaux cxéculés denuis 1897 ot je reconnais que les résultals obtentus ne sont pas à dédaigner, mais ils ne sont rien, à mon sens, en comparaison de ce quil reste a fare. Ces projels, au nombre de deux ou trois conts pour toute la France, ne représentent qu'tune gbutte d'eau dans l'océant.

Ce que jo demande aujourd'hui c'est un plan d'ensembie pour nos départements do montagnes.

Je demande que l'on opère non pas seulement sur qudques poinls particuliers, le Cantal on los Pyrenées, mais sur" la lolalité du pays.

Malheureusement, ce boau rêve est actuellement absolument irréalisable, en raison de l'insurfisance des crédils affectés à ces travaux. C'est ainsi que jarrive à ma conclusion.

Quand le service a été constilué, le crédit primilif qui Iui a élé altribué était - jose le dire - infinjtésimal : 30.000 francs seulement. C'était un simple crédil d'amorce, une manjère d'encourager les communes, de leur faire savoil que ce service existaif du minthtère de l'agriculture, qu'il élait d̀ leur disposition et qu'il pouvait Jeur accorder des subventions.

Ce pelit crédil de 30.000 francs a suff pendant les premières années. Mais ensulite, au lieu de l'augmenter, on n'a pas cossé de le léduire el, si je ne me trompe, il a clé un certain moment qélduit à $15.000 \mathrm{fr}$, ce qui était déplora. ble.

M. le ministre Ruau comprenant qu'il élail impossible d'obtenir des résullals séricux avec de sil faibles ressourcos, l'a fail rélablir d'abond à son chiffre premier, ot il a même eu le courago d'mprunter, à un autre chapitre, of qu'il jugeait nécessaire noul pousser plus entierement les travaux. C'est ainsi qu'en 1905, on a pu disposer d'une somme de 70.000 francs, et en 1906, d'une somme encore plus con* siderable.

Je félicitc M. le minisho de son initialive el je l'en te. mercie

Malgrré tout, la situation n'est pas nette; elle niest pas définte: : je n'ose pas dire qu'elle n'est pas réguliere.

Il faul que ce service soit doté par lui-mème, il ne faut pas qu'il soit obligé d'emprunter" aux differents chapitnes du budget ce qui luil esl nécessaire pour vivre.

M. le ministre de lagriculture a fail un emprunt au ctes. dil du chapitre que nous discutons ; mais la loi de 1882, je lai dil tout à l'heure, s'applique surtout à des cas spretaux : jc sais lres bien qu'cle a aflecte unc petile somme aux améhorations pastorales mais d'abord cette somme est tout a

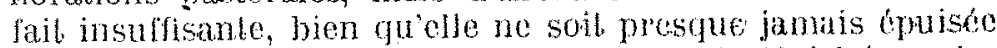
par ladministration ; puis, dans la pensée du législateur, les améliorations nastorales qu'il a ainsi autorisés se lient élroilement aux travaux speciaux prévus par la loi de 1882.

En réalite, le scrvice pastoral n'est pas doté comme il devrait l'être; il faut Iui donner des crédils sulfisants pour arriver à des résultats ; ces crédits ne sonl pas seulement nécessaires pour lexécution des travaux, ils doivent pourvoir aussi aux besoins du personnel.

Si l'on veul conduire partout la vaste opération qu'il s'agit d'entreprendre, il sera sans doute nécessaite d'augmenter un peu le nombre des agents forestiers ; ll laudra, par exemple, avoir des agents régionaux pour traiter avec les communes, diriger les travaux of surveiller les agents locaux. Ce ne sera pas une grosse dépense, mais ce sem une dépense. 
Comment arriver à doter convenablement ce chapítre nouvoru ? Je ne me berce pas de l'illusion que, dans notre siluation financière, nous obtiendrons aisémenl un gros relèvement de crédit, mème pour une ceuvre aussi utile. Les 175.000 francs proposés à la Chambre des dépulétes, et repoussés, seraient bien insuffisants; qui pourrait cependant nous les garantir pour l'année prochaine ? Les mêmes objections se représenteront certainement. Dans ces condition, je ne vois plus qu'une mesure à prendre : c'est de modifier le chapitre sur lequel vous délibérez de le diviser en deux parties.

Il est de 3.375 .000 francs. Ces 3.375 .000 francs sont con. sacrés, d'après la loi de 1882, aux travaux exceptionnels, dont je vous parlais tout à l'heure, à des travaux d'ar't et à des acquisitions de terrains. Qu'il soit entenđư, dût-on pour cela reviser la loi - ce qui sera ma conclusion - qu'on pourra prélever désormais sur ce chapitne, au fur el à mesure des besoins du service, ce qui sera nécessaire pour les opérations pastorales qu'on doit entreprendre dans toutes les parties de la France. On dépensera 200.000 francs en une première annéte, 300.000 francs ensuite, 500.000 francs plus tard, ol j'espère qu'un jour" viendra où le Parlement dans sa générosité, voyant que l'emploi de ces fonds est créateur de richesse, se décidera à donner de l'argent, de l'argent bien employé, qu'il retrouvera au centuple par les plus-valuca d'impóts dans le budget lui-mème.

Je le rệpètc, à l'heure acluelle, il n'est pas possible daccomplir" la grande oeuvre qui s'impose sans inscrire un, nouveau crédit au budgel, et peut-êlre sans roviser la loi de 1882, car cette loi n'est pas intangible, elle l'est moins que tout autre.

J'en ai fini. Je recommande ces observations à l'attention et à la sollicitude de M. le ministre de l'agriculture. Je ne lui demande qu'unc chose, c'est d'étudiler la question de façon à pouvoir nous apporter, lors de la prochaine discussion du budget, des propositions fermes sur ce point. Il connaîl trọ bien la quesion, il est trop dévoué à nos populations des montagnes, au milieu desquelles il vit, pour que je n'aie pas pleine conflance dans la solution qu'il trouvera.

\section{DISCOURS DE M. AUDIFFRED}

Messicurs, je demando au Sénal la permission d'ajouter quelques observations aux considérations si probantes et si ólocuenles que l'honorable M. Méline vient de développes levant vous. Comme M'. Méline, je signale à l'attention: du Sénat l'insurfisance du crédilt consacré au reboisement.

Il élail, il y a quelques annéas, de 3 millions 500.000 francs, il a été réduit à 3.250 .000 francs on 1904 , et on vient de le relever à 3.375 .000 francs. Si l'on continue à procéder" avec celle lenteur, on peul être convaincu que l'œuvre du reboisement et du gazonnement ne s'accomplira pas en un siè('lc ! Il y a là véritablement un grand danger sur" leque] h. Méline s'est expliqué, et que le Sénal oomprend.

Jo crois que, pour réussir à menè à bien celbe vaste rnlreprise, il faut absolument avoir les populations avec $\therefore$ i, que l'on ne peul pas songer à reboiser les montagnes si les populations de ces pays n'y consentent et n'y prêtient lour concours le plus actif. Or, par suite de cirtconstances diverses, et, par suite de certaines fautes, que M. Méline andiquerer, et sur lesquelles je ne veux pas insister, on a peut-être eu tort de ne s'occupen" d'abord que du reboisement.

Il aurait fallu accorder un avantage aux populations de montagnes, et cet avantage, c'est l'amélioration de leurs pâturages. Jo crois qu'il faut faire marcher de pair la transformation en prairies productives des terrains incultes, et les reboisements de certaines parties.

On affrme que la chase est tout à fail difficile ; M. Méline me rapportait hier qu'on allail opposer à la demande qu'il a formulée une objection basée sur le mauvais vouloir des populations; et comme j'ai été mêlé très directement à cette question, je demande au Sénat la permission de lui dire en quelqueis mots ce qui s'est passé.
Dans la séance de la Chambres des députés du 4 décembi'e 1900, j'ai demandé à M. Ie ministre de l'agriculture c'étail alors M. Jean Dupuy auprès duquel j'ai toujours trouvé pour les questions d'intérêt général le concours le plus empressé - de vouloir bien faire procéder à des études de reboisement dans le plateau supérieur qui domane la vallée de la Loirne.

M. Jean Dupuy voulut bien me dire qu'il était complètement d'accord avec moi, et il nomma immédiatement un agent chargé de l'étude de ces périmètres.

Ces études aboutirent à la constitution de diverns projets qui furent soumis aux intéressés.

Dans le département de la Loire, toute la région comprise entre Rive-de-Gier et Saint-Chamond, qui est complètement dénudée - on s'ens rend compte en allant par chemin de fer de Saint-Elienne à Lyon - accepta avec empressement les conclusions des forestiers ; mais toute la partie confinant à l'Auvergne protesta au contraire.

Comme membre de la commission chargée a"examiner les réclamations, et dont faisaient partie l'inspecteur des forêts, l'ingénieur" or"dinaire, un conseiller d'arrondissement, je dis aux intéressés : Vous protestez; cependant, ce que nous désirons entreprendre est dans votre intérêt. Voulez-vous que nous allions sur place nous rendre compte des objections que vous formulez?

Après un voyage de cleux jours, nous anrivàmes dans une communo extrèmement inléressante, Cllalmazelles, située à la limite de la Loire et de l'Auvergne.

Là, j'ai vu un spectacle que je n'oublierai pas, une étendue de 900 heclares sans al’res, à peu près. sanı herbe, envahie prosque complètement par la bruyère. Une réfiexion vint à l'esprit de lous les visiteur's : Comment, dans ces bruyèress, le bétail pouvait-il trouver" un aliment?

Et je remarquai que, sur certains points où les animaux avaient enlevé les bruyères en passant fréquemment, poussait une herbe tròs drue, treès scrrée, tout à fait nourrissante sous l'achion de l'eau qui coulait partout sur ces hauleur's. Je fis alors aux habitants de celte commune une pror position conciliante : "Si vous le voulez, nous allons conclure une convention ; nous prendrons l'engagement de ne pas parler de reboisement tant que nous n'aurons pas amélioré vos prajries, de telle façon. qu'elles vous permiettent de nourrir" cent tètes de bélail dé plus ".

IIs acceptèrent avac empressement, nous avons voté 'à l'unanimité la résolution qui accompagnait cet accor'd et depuis j'ai poursuivi la campagne que j'avais entreprise.

Faisant partie de la commission du Touring-Club, qui s'occupe du gazonnement et du reboisement avec le zèle et l'intelligence que l'on sait, j'ai prié les éminents prélsidenls, M. Ballif, et vice-président, M. Doffer, de cetto grande association, qui était disposée à fajre des sacrifices poun lo reboisement et lo gazonnement, de vouloir bien accorder une des subventions de l'association au département de la Loir"e. Nous sommes allés trouver ensuiter $M$. le ministre de lagricultule, et le $\mathrm{M}$. le direcleur des lorêts, et nous leur avons proposé, si l'Ftat voulail donner' $2.500 \mathrm{fr}$., de clonner", au nom du 'Touring-Club, $2.500 \mathrm{fr}$. pour tenter une expérience damélioration pastorale.

Celte expérience devait avoir lieu dans la commune de Chalmazelles.

Or il est arrivé, pour les raisons que M. Méline vous indiquait tout à l'heure, que celte commune a refusé ce cadeau.

Que voulez-vous ? Il y a des préjugés très vivacesi, très invétérés, trop souvent justifiés par' des actes anciens d'immixtion abusive de l'Flat ; mais ils ne dureront pas toujours. Je me rappelle qu'il y a vingt-cing ans, quand nous poursuivions dans la Loire l'œuvre de la vicinalité, quand nous voulions doter les communes qui n'en avaient pas encore de ces voies de communication qu'elles apprécient si bien maintenant, je me suis heurlé à des oppositions encore bien 
plus inacceptables. Dans deux communes, je n'ai pas pu réussir" à lairé accepter" une subvention de 40.000 francs consentie pour" chacune d'elles, sous l'unique condition de réaliser a la caisse des chemins vicinaux un emprunt de 4.000 francs à 4 pour 100, pour lequel elles aurajent paiyé 160 fr. par an seulement pendant trente ans.

Ces mèmes communes, autrefois si rélractaires à l'œuvre de la vicinalilé, qui la repoussaient même, quand on leur apportait des monceaux d'or, exécutent ajourd'hui tous les chemins qu'on leur" propose; et contribuent pour" le tiers à leur exécution.

J'estime qu'il en sera pour le gazonnement et le reboisement comme il $\in$ n a été pour la vicinalité. Mais, monsieur le ministre, et messieurs les agents des forèts, il faudra beaucoup de tomps, le palience et de persévérance pour aboulir ; il faudra vaincre les préjugés, disculer avea ces braves gons ; il faudra leur faire comprendre que c'est leur intérêt qu'on poursuil, en mème temps que l'intétêt général. Il faudra commencer' par leur rendre des services de nature à être appréciés par eux. Il faudra créer par exemple des établissoments comme le crédit agricole dont ils ont grand besoin; il faudra savoir quelles sont les choses qui peuvent leur être avanlageuses, of les leur donner. En un mot, il faudra s'ingénier pour los gagner à cette cause du gazonnement et du reboisement.

Messieur's, nous avons en france quelque chose comme 9 millions. d'hectares inculles, sur" une sualace tolale de 520 millions. Les hommes les plus compétents de l'adminstration des forêts sont d'accord pour reconnaître qu'on pourrait utilement constituer des prairies sur 3 millions d'hectares. Et, messieurs, si nous arrivons à convertir en prairies assez fertiles 3 millions d'hectares incultes, en comptant un revenu de $40 \mathrm{fr}$. par hectare, nous réaliserions anmuellement une augmentation de produits, dans ce pays, de 120 millions. Si nous parvenions, par une couvre de longue haleine, à convertir les 6 aulres millions dhectares, en forêts, nous aurions un autre revenu de 240 millions à raison de 40 francs par hectare. 120 millions pour les prairies, 240 millions pour les forêts ; nous augmenterions donc la production annuelle dans les pays de montagne de 360 millions par an, sur quoi le fisc prélèverait son tribut ordinaire, c'esta-dire onviron un septième, soit 50 millions. Cette ressource lui viendluait, sans qu'il eût à aggraver les charges, en enrichissant au contraire les conlribuab]es.

Je crois que le fisc devrait, dans ces conditions, contribuer à ces dépenses de gazonmement et de reboisement. II le pourrait d'autant pilus que le: non-gazonnement et le déboisement causent un piréjudice considérable chaque année aux populations des vallées.

on a constalé depuis cent cinquante ans, dans la vallée de la Loire, vingt-six inondations, ch chacune à coûté 100 millions. Quand une inondation: se produit, ce ne sont pas seulement les populations qui sont éprouvées, c'est l'Etat, car il est sollicité de leur venir en aide, et il paye des indemnités ef des secours imporlants qui grèvenl considérablement son: budget. Il vaudrait assurément mieux prérenir que réparer.

Je voudrais vous donner, par" une indication sommaire, une idée du régime de certains cours d'eau.

Voilà la Loire, dont le bassin occupe à peu près le cin quième de notre territoire. Savez-vous, adors que la rivièro de la Somme n'augmente que de quatre fois le volume en temps de crue, que les crues de la Seine n'augmentent le débit du fleuve que do trente fois, saver-vous de combien est augmenté le débit de la Loire par le fait des inondations? A Roanne, le débit, d̀ l'étjage, est de 8 mètres cubes a la seconde et, en temps d'inondation, il arrive à 7.000 mètres cubes, c'està-dire neut cent fois plus. Vous pouvez penser ce qu'est ce torr'ent dévastnteur quand de pareilles éventualités se produisent.

Au Bec-d'Allier, la Loire angmente encore de sept cent fois son volume, à Tours, de trois cont fois. Il en résulte que les roches sont entrainéos, transformées en galets, puis en bancs de sable, el que lorsqu'on passe à Nevers, a Orléans, à Tours, on ne voit pas un fleuve, mais un Sahnta selon l'expression d'un conservateur" des forèts.

C'est la destruction, el il laul éviler cette destruction. Ces forces de destruction, il faul les convertir en forces créatrices ; il lant leur faire produire des résultats utiles au lieu de les laisser se dépenser en dévastations.

Il y a un moyen, c'est colui que les forestiors connaissm, celui qu'ils ont si admirablement réussi à pratiquer" : c'est de placer sur celte vaste étendue de rochers, de terres nucs, soit du gazon, soit des [orêts. Alors le gazon, l'humus de la loret, procèdent par voie d'évaporation, of aussi et surlout, à la façon de l'épongc, absorbant des quantités énormes deau, restituées ensuitc soit sous formo de sources, de ruisseaux coulant constamment, soil sous forme de forces hydrauliquess.

Vous avez créé des canaux, amélioré le lit de certaines rivières, il importe de régulariser leur débit dans l'intérêt de la navigation qui pourrait etre compromise, si l'on n'avisait.

Voilà, messieursh l'opération à faire, eller est vaste, considérable, immense, mais faut-il pour cela fermer las yeux ? faut-il attendre que les dévastations soient consommées sur un plus grand nombre de points, ef ne vaut-il pas mieux les prévenir et, dès à présent, s’ingénier à améliorer" cet élat de choses vr"aiment dúsastreux ? Voila la question que le Sénat et la Chambre doivent aborder avec la rúsolution daboulir à un résullat utile.

L'Etat croit faire des économies quand il réduit le crédit à 3.500 .000 francs ; lout au contraire, il porte un prójudice énorme à son budget des recettes. C'est à tort qu'il réduit ces dépenses ; il devrait les augmenter.

Je comprends que, dans l'état actuel de nos finances, il soil très difficile de demander des augmentations, Cependant je voudrais, ayant écoulé avec beaucoup de soin l'admirable discour's de M. Poincaré, qui esł un éminent nnancier, faire cetlo simple remarque que, en général,' les fnanciler's les plus habiles sont trop tentés de ne se préoccuper ruer de deux choses : réduire les dépenses et amortir. Ils ont raison, cela est excellent, mais il y a autre chose à faire : c'est de créer" la richesse, c'est d'augmenter la production. Alors seuIement le Trésor trouverait, sans création d'impôts nouveaux, sans augmentation des impots existants, dest ressources qui lui viendraient naturellement d'un pays plus riche el plus capable de satisfaire la soif insatiable du Trésor.

Jusqu'à présent, on n'a malheureusemenl vu dans le budgel qu'une machine financière. Je crois qu'il faub y voir autre chose. Je crois qu'il faut ètre d'une sévérité plus rigoureuse qu'on ne l'a été dans le passé pour des dépenses qui, en somme, ne produisent rien. Mais je crois cu'il fout elre plus large, sans compromeltre l'équilibre finanier - - je ne voux d'excès on rien - pour les dépenses qui ont pour résultat d'empêcher des destructions et de créer des ressources nouvelles pour le pays el pour le Trésor.

Ceci dil sur le rôle de I'Etat, sur la nécessité qu'il y a pour lui à intervenir" plus largement dans ces dépenses, j"estime qu'il peut trouver à colé do lui des concours précieux, qu'il n'a pas recherché jusqu'ici.

Dans le service des forêts on compte, comme dans toutes les grandes administrations de l'Etat, des bénédictins, qui savent exécuter des cuvres de grande portée. Un inspecteur général des forêts, aujourdhui en retraite, M. Mélard, a fait un travail duquel il résulte que, dans le monde entier, on consomme annuellement pour 250 millions de bois d'œuvre de plus que n'en produisent les forêts, et que l'on epuise hativement ces forêts constituées par les siècles. M. Mélard a soumis son travail, lors de l'Exposition universelle, à tous les forestiers du monde, venus pour assister à cette grande manifestation, et tous lui ont déclaré quil avait non seule- 
ment dil la vérité, mais qu'il étail resté au-descous de la vérilé.

Eh bien, massieurs, nous avons en France me silualion relativement privilégiée. Alors que l'Allemagne achète pour 350 millions de bois a l'etranger de plus qu'elle n'en produil, compant à blanc, en ce moment, cortaines de nos forêts, alors que l'Angleterre achete 500 millions de bois de plas qu'elle ne produit, la France, elle, n'achele que pour 110 millions de plus qu'elle ne produit. J'estime que, ces 110 millions de bois, elle peut les produire elle-mème et livier encore à l'exportation pareille quantité.

Voilà le problème à t"ésoudre.

Ceci oxposé, comment arriver à trouver, pour les forestiers, ces resisources qui leur sont indispensables ? Il ne convient pas de s'adresser uniquement a l'Etat.

Voici pourquoi.

Messicurs, il y a quolques années, les compagnies do mines payaiont le mètre oube de bois 16 francs ; un reu plus tard, 21 Irancs, et, avant d'entrer en séance, l'un des com missaires du Gouvernement m'a affrmé que le prix achuel variait de 20 a 25 franes.

Il mia encore déclaré que sur" le marché de l'Europe centrale le prix du chene avait augmenté cette année de: 60 pour 100 , et que le prix de la résines avait doublé en. France comme ailleurs.

Les seules mines de la Loire achelent pour 5 millions de bois anivellement ; toules leis mines de France en consomment pour 500 millions.

Si le bois d'œuvre a subi ces augmentalions, il en résulte. un surcroil de charges considérables pour les consommar teurs. Quel est donc, dès lors, l'intérêt de ces sociétés ninières?

N'est-ce pas de contribuer à la restauration du domaine forestier avec l'aide du Gouvemement.

Voilà une premjère catégorie de personnes auxquelles le Gouvernement peut s'adresser.

Mais les villes qui essayent de pourvoir à leur alimentation en eau potable au moven de grands barrages, comme Sint-Elisenne, Roanne, Rive-de-Giefr, Saint-Chamond, Firminy et tant d'autres, ne doivent-elles pas élendre le rayon des forêts autour de leurs réservoirs ?

A Saint-Eutienne, par exemple, où les barrages retiennent 6 à 7 millions de mètres cubes, nous avons vu cette année la population sur le point de manquer d'eau. Dans les réservoirs, il n'y avait plus, à un moment donné, qué 125.000 mètres cubes, cest-à-dire de quod pourvoir à une consommation d'une semaine.

Des villes comme Saint-Etienne, comme Roanne, Rivede-Gier, Annonay, etc., ont donc intérêt à reboiser dans lc rajon d'alimentation de leur barrage.

Il en est de même pour les compagnies de chemins de fer qui payent, chaque année, pour leurs traverses, leurs wagons, des sommes énormes. Elles aussi, nontelles pas pour devoir de venir en aide à l'Elat ? N'éviteraient-elles pas ainsi le renchérissement de ces bois d'cuvre ?

Il est aussi une autre catégorie de collectivités puissantes qui peuvent également intervenir avec utilité pour la reconstitution ou plutôt la conslibulion du domaine forestier de ce pays : ce sont les institutions de prévoyance.

Dans notre pays de France; où nous sommes très prudents, où, sous des apparences quelquefois contraires, nous sommes très peu novateurs, nous n'admetlons pas, jusqu'ici, qu'un placement puisse présenter toute sécurité, en dehors de listat. C'est dans les caísses publiques que les capitaux des travailleurs modestes viennent s'engouffer.

C'est ainsi que les caisses d'épargne ont 4 milliarù à la caisse des dépôts et consignations ; que la caisse nationale des retraites possède 1.500 millions ; que les sociétes de secours mutuels possèdent un capital de 500 millions, remis à l'Etat; et que les sociétés comme les Prévoyants de l'Avenir, comme la Boule de neige, pretent, elles aussi, lear avoir dune centaine de millions aux départements el aux communes.

On arrive ainsi à une somme lotale d'environ, 6 milliards. Serail-il exessil de temander qu'uno parlie de ces capitaux -.. Je ne dis pas uno partje importante mais la inilieme partic seulemont - soil affectée, charqua anné, comme cela se passe dans les pays etrangers, pour les construclions dhabitations a bon marché, el notamment de prets agricoles, au développemenl de notue richesse publique ? Ne serait-il pas sage de faire refluer une partie de ces capitaux vers les populations qui les onb épargnné, pour les faire servir à l'amélioration de leur bien-être ?

M. Lintuhac. - Très hien. Mais ajoutez-y l'apostolat des professeurs d'agricullure.

M. Audifrred. - Je crois que si M. le ministre de l'agriculture voulait bien constituer une commission pour étudier ce problème que jai esssayé d'exposer au. Sénal, il ferait nuvire utile.

Je suis convaincu que celle idée de tout confer à l'Etat, loute l'épargne des plus numbles, pour qu'il la place en valeur's cst une idée qui, poussée à l'excès, a fait son temps.

Je crois que si $M$. le ministre de l'agriculture voulait bien constituer une commission composée de ses inspecteurs des forèts dont la haute valeur ast universellement roconnue, de quelques professeurs d'agricullur"e, puisque M. Lintilhac a bien voulu rappeler, ce qui est vrai, qu'ils onl été en celte ceuvre des apotres, de financiers éminents qui ont le devoir, qu'ils remplissent, de ne jamais laisser dépenser de l'argent sans lui assurer une grande sécurité, si M. le minishe voulah bien ajouter encore à celte commission quelquesunes de ces personnes très connues, qui, depuis trente ans, ont élucidé avec lant d'intelligence tous les problèmes de la prévoyance sociale, jet crois que l'année prochaine nous pourlions peut-ètre ulilement reprend"e ce débat, el réaliser des solutions que la commission elle-meme aurait probablement proposées.

\section{DISCOURS DE M. FLAISSIÈRES}

Messieurs, je ne viens pas seulement donner mon assentiment public aux discours si suggeslifs et si précis qui on clé prononcés avant moi à cetle lribune; je me serais borné, ce que j'ai fait, d'ailleurs, à donner mon assentiment particulier el à applaudir - ce qui n'est point banal d'ailleurs notre éminent collègue M. Méline. Mas si je ne communie point dans la mème foi politique et économilque que l'honorable M. Méline, je communie dans son amour de la terre, que je parlage avec lui, et je m'intéresse très vivement à toules les questions agricoles.

C'est, monsieur le ministre de l'agriculture, pour vous signaler deux points spéciaux, dans cette étude générale, que j'al pris la liberté d'aborder cette tribune, ce dont je m'excuse d'ailleurss devant le Sénat.

Le ministre de lagnticulture, et la majorité du Sénat, s'intéressent à la reconslitution des bois ef à la restauration des montagnes, à leur conservation. Depuis quelques années, j'assicte, avec le plus grand interêt dans la région du sud-est. de la. France, notamment dans le département des BassesAlpes, à des lentatives vraiment remarquables de reboisement. J'y ai vu et vous y verrerz avec quelle ténacité lounble, j"allais dire quel acharnement, l'administration des foiets procede pour reconstituer des forêts ou if n'y a aujour. d'hui que des rochers absolument incultes, qui, par' leur déclivité, leur nudité absolue menacent la plaine et permet. tent toutes les inondations dont on se plaint si justement!

Il y a peut-être quielque chose de mieux a faire, messieurs, que la eréation de forèts nouvelles en remplacement de celles qui ont existé, et c'est plus spécialement le fait que j’ai l'honneur de signaler à M. le: ministre.

Je vais meltre en cause des personnalités morales sur les. quelles le Gouvernement, n'a pas une action aussi directe, 
aussi efficace, aussi ulile qu'elle peut l'ètre lorsqu'il s'agit des personnalités morales telles que les communes ou les départements.

Je mets en cause plus spécialement les propriétaires euxmêmes, et je lais appel aux souvenirs, à la douleur", de lous nos collègues, qui représentent ici des pays où l'on cullive le châtaignier, pour signaler à $M$. le ministre l'action vérł. tablement funeste ef sauvage, exercée aujourd'hui, contre les forêts de châtaigniers par les acheteurs qui livront à l'industrie de fabricalion du lannin, les spécimens les rius beaux et les plus jeunes de ces arbres.

Monsieur: le ministre, il y a là un double drnger. Quand on abat un chêne, ou un ormeau, evidemment or nuit à la forèt que l'on dépeuple ainsi, alors qu'on doviai'a replanter. Quand on coupe un chataignier dans sa maturité, dans sa vigueur et dans sa jeunesse, non seulement on déborise, mais on diminue la valeur du sol ou poussait ce chataignier" et sur le schiste duquel il peut. seul produdire une appréciable récolte ; on met en péril l'alimentation humaine ainsi que l'élevage des animaux.

C'est ainsi que l'altenlion du Gouvernement peut etre, je crois, utlement attirée sur cette quesion.

Je sais bien que les moyens de persuasion qui vous étaient lout à l'heure indiqués et conseillés, pour ce qui a trait aux communes et aux départements, auraient peutêtre encore moins de chances d'aboutir auprès des particuliers, qui ne sont point toujours dans l'opulence dans les régions de la culture du châtaignier. Mais, tout à l'heure, notre éminent collègue, $M$. Audiffred, vous demandait daugmenter la dotation de certains services; ce serait le cas, monsieur le ministre, et, assurément, ce ne serail qu'tune avance que vous feriez au pays par. voie de primes. On en donne bilen à d'autres branches de l'agriculture !

Par voie de primes, peut-ête pourriez-vons oblenir des propriétaires qu'ils ne se laissent pas nller à se dépouiller eux-mèmes, car ils sont les premières victimes et dévorent leur blé en herbe; peut-être pourriez-vous les amener à résister aux offres alléchantes qui leur sont faites par les marchands de ce bois précieux, el qui ne tardera pas à disparaìtre devant cetlei dévastation.

Pour ce qui a trait au reboisement velillez me permettre, monsieur le ministre, lout en alfrmant que je suls comme tous les amis de l'agriculture et des arbres, partisan de la restauration des forêts, de leur reboisement, de leur conservation, veuillez.me permettre de faire une réserve modérée pour ce qui a trait au reboisement dars certaines régions, de me faire l'écho' des plaintes qui ont été formulées par la culture ef rindustrie pastorale des Bouches-du-Rhone et notamment des immensitég du désert de la. Crau el du pays d'Arles ( $\left.{ }^{*}\right)$.

Vous savez, Messieuns, que les troupeaux transhument pendant la période de juillet, jusqu'en octobre ou décembre vers les paturages des Hautes-Alpes. Ils ont vécu pendant les mois d'hiver el du printemps dans les Bouches-du-Rhone et dans la Cnau ; ils vont continuer a vivre dans les HautesAlpes pendant la période de chaleur ét de sécheresse.

Eh bien, l'administration des forêts a peut-être outrepassé le but qu'il s'agissait de poursuivre et, pour assurer, dans une période qui ne serait assurément pas moindre de cinquante ans, puisqu'il faudrait ce temps pour permeltre à uno forèt de se développer, pour assurer le paturage à une période indéterminée, aujour'dhui, on compromel le pâturage actuel, en poussant jusqu'au bout la reconstitution des forèts et en voulant quand mème restaurer, reboiser" les hauts pla-

(*) Nous nous associons pleinement aux regrets manifestés par l'orateur sur la destruction des foréts de chrtaigniers, et nous esperons que les progrès de l'industrie permettront bientôt aux fanneurs de remplacer exclusivement le tannin. meurtrier des forets, par le chrôme, produit inerte du sous-sol. Mais nous faisons toutes réserves sur la suite du discours de M. Flaissieres. teau des Alpes sur lesquels vont paturer les troupeaux des Bouches-du-Rhone et qu'on verut en éloiguer.

si, et cela n'est point dontenx, le teboisement cst absolument nécessaire dans les parties déclives dos monlagnes, il devient beaucoup moms indispensables, sur les hauls plateaux où il n'y a pas de dénivelloment très considérable, ot où les eaux ne peuvent, en aucune façon causer les ravages qu'elles occasiomnent lorsque la partie esl déclive.

Je suis convaincu qu'il maura suffi de vous signaler le fail pour que, dans votre clairvoyance, monsieu" le ministre, vous donnier les ordres nécessaires afin que votre service n'aille pas plus loin qu'il no convient dans lamoup qui nous est commun de l'agriculture, des forets et de l'elevage!

\section{DISCOURS DU MINISTRE DE L'AGRICULTURE}

Messieurs, l'honorable M. Méline, dans les observalions de portée générale qu'il a présentées au sénat, a posé devant l'Assemblée le probleme de la conservation de la monlagne. Il a rappelé qu'étant ministre de l'agriculture il avait crée le service des amélioralions pastorales. Les crédits dont dispor sait la nouvelle institution: étaient extrèmement restreints, mais elle élail dirigée par un homme de valeur, qui a fail ses preuves.

Si, en 1898, au lendemain mêmo de celle crénlion, le nombree des projels mis à exécution élail de 4, l'entreprise d'amélioralions pastornales a fait lant de progrès qu'il est aujourd'hui de 184 el que si, en 1898, M. Méline dépensail. $1.358 \mathrm{fr}$. pour ces travaux, nous ayons pu, lans les deux dernière. années, dépenser 56.000 et 60.000 francs. Néanmoins ces crédits sont tout à faili insuffsants. Sans doute la loil do 1882 sur la. restauralion des terrains en montagne a permis l'ex. binction de beaucoup de lorrents, elle a foumi le moven d'exéculer de très nombreux travaux qui sont, malheureusement, plulòt des onvrages d'ingéniours que des travaux formstiers proprement dits.

Mais depuis celte epoque, ot malgré ces travaux, de nombreuses calastrophes se sont produiles. Je rappellerai au Sénat les inondations de 1897 dians les Pyrénées, tes ratastrophes de Bozel, de Fourneaux el d'Ouzous, qui ont prolondémenti ému lopinion publique.

Si des désastres semblables onl pu bouleverser une région, si linsulfisance de protection éclate manifestement encore sur bien dest points, c'est quo l'ouvre du reboisement n'a pas éte loujours acoueillie comme olle aurait dh l'ête par les populations des hats plateaux. Aussi suis-je bien pres de parlager' l'avis de M. Méline, ef de reconnatre qu'on leur eût mieux lait accopter les reboisements en les faisant procéder par des amendemonts ou des extensions des paturages.

De meme que le ministere do lagyicullura, delats quelques

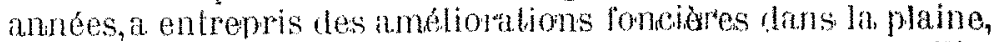
de meme il serail ulile que l'on fit on monlagne des ameliorations do menos matulto, qui próserveraien! hien souverd la vallée las désastres que lui réservenl les flarros abrupts el dé. nudés des haules dimes.

Ces travaux seraient de peu d'importance ot couleraient fort peu. Une bonne ententio enl,re les services forestiers et les communes les faciliterait.

Sans doute les communes craignont que lour propriélés soit soumise au régime forestier of au cortege de petites vexalions que ce réfgime endrathe.

Mais jo dois reconnailro, of je le fais avos lo plus grand plaisir", que l'administration forestiere, dans sa délicate mission, sest fait aimor des populations, et qu'aujourd'hui, il u'existe plus de ces conflits si frequents aubrefois.

C'est alors que tout naturellement cet apostolat auquel on faisail allusion tout a theure - on pourrait dire plus modeslement ce service d'ensoignement - doit trouver sa place. Presque tous les agents forestiers, dans les regions de montagne, on lait comprendre aux populations le bénéflce qu'elles retireraient, soit de la conservation de leurs paturages, 
soit de l'établissement de chemins d'accès, d'abreuvoirs, de cabanes-abris, de débrousaillements, qui constituent les modestes travaux d'améliorations pastorales

on accomplirait évidemment une ouvre profitable, productrice a la fois de sécurilé et de richesse, en employant a ces améliorations les crédits que la loi de 1882 alloue annuellement pour la restauration des terrains en montagne.

Jai pu, grâce à une ventilation très légitime, augmenter, mais dans une proportion bien trop faible encores les cré dits affectés aux améliormations pastor'ales. J'espère que sans être obligé dé recourir à une transformalion de la loil de 1882, je pourrai, dans l'avenir, leur réserver" de plus fortes sommes encore.

Je m'engage, sans faire appel à de nouveaux crédits, à demandor au service forestier de m'adresser, sous forme de rapports, l'ensemble des améliorations pastorales qu'il croira ulile d'exécuter" dans le pays, ce faisant, nous arriverons, je l'espère, à maintenir sur" les pentes dénudées, sur" les rochesi incultes, ce gazon quion qualiflait d'herbe éponge et qui, peut-être mieux que le reboisement, prévient les inondations.

Messieurs, cette restauration del la végétation ne présente pas seulement un intérêt réel pour la protection contre les catastrophes, mais elle aura aussi pour conséquencer de régulariser le débit des sources. Il est reconnu en effet que, pour des causes qui échappent encore, les sources se font un peu plus rar"es, el que leur débit diminue d'année en année. On étudie, au minislère de l'agriculture, dans certaines commissions tochniques, le moyen de remédier à cet appauvrisisement. Les indications foumies par le service forestier pourront nous montrer quels remèdes seront les plus utiles pour empêcher le tarissement de cette eau qui fait aujourd'hui la vie, l'énergie et la lumière.

M. Audiffred me permeltra de lui dire que, à ce point de vue, nous avons fait tout le possible dans le département de la Loire. Il a bien voulu reconnaître que l'honorable M. Jean Dupuy, mon prédécesseur, avait aceordé à ce département un agent forestier spécial. Il sait que depuis que je suis au ministère de l'agriculture, j'en ai fait autant et que je me propose de continuer ce service.

Nous avons déterminé un certain nombre de périmètres de theboisement dans le bassin de la Haute-Loire. Nous avong même fait mieux, M. Audiffred le rappelait tout à l'heure. Nous avons cherché une entente, diaccord avec le conseil général de la Loire, le Touring-Club, et le ministère de l'agriculture, pour que l'on fasse un essai sur les hauls plateanx de la commune de Chalmazel.

Il s'agissait de mettre en valeur environ 500 hectares de terrains qui ne produisent presque rien car ils ne rapportent, d'après les renseignements fournis, que 75 centimes par hectare.

La commune de Chalmazel, pax une sorte de méfiance que rien ne fait comprendre, car on ne lastreignait a aucune espèce de condition, on ne dérangeait en rien le régime de ses sources, quoi qu'on en ait prétendu, a refusé ; on lui offrail $5.000 \mathrm{fr}^{*}$. pour faire une sorte de champ d'expérience qui aurait permis, d'après les évaluations, de porter le rendoment de 75 centimes à 15 francs; ellé les a repoussés. Elle s'est confnée dans une espèce de ménance et de routine, avec lequel tout progrès devient impossible.

Je désire, messieurs, ménager les instants du Sénąt. Aussi me permettra-t-il de ne répondre que brièvement a $\vec{M}$. Flais. sières sur deux points.

Lhonorable sénateur m'a fait remarquer" que la destruction des châtaigniers était une calamité. Je partago absolument son avis.

Je me souviens des belles paroles de Michelet sur ces arbres qui, comme des palriarches, font à la forêt une entrée vénérable et dont les feuilles si vertes do vie, étendues comme des mains, cherchent la Iumiere et s'en imbibent avidement.
Lo châtaignier n'est pas seulement un très bel an'bre, c'est surtont un arbre utile, parce qu'il produit un bois d'cuvre excellent, un bois de construction el en même temps parce que son fruit nourrit l'homme et les animaix.

Tous les fléaux se sont abattus sur lui. D'abord la maladie. Nous avons cherché à découvrir d'où provenait le mal, et des études très savantes ont étés poursuivies au ministère de l'agriculture depuis quelques années. Je rappellerai les beaux travaux de MMI. Prunet et Mangin: et de M. Grillez, mais nous ne sommes malheureusement arrivés encore à aucun résullat.

D'autre part, les fortêts de jeunes chataignier's sont l'objet des convoitises de certains marchands de tanin. On détruit les forêts de châtaignivers, et on les détruit en masse.

Nous avons également recherché s’il existait un arbre qui lui futt équivalent : nous ne l'avons pas encore trouvé ; nous emploierons donc, soyez-en persuadé, tous les moyens qui sont à notre dispositions, pour nous opposer à la mort ế à la disparition du châtaignier.

M. Flaissières nous a parlé, d'autre part, des reboisements à outrance ; il nous a dit que dans certaines régíons, on reboisait d'une façon véritablement immodérée. Il y a peut-être là quelque exagération. La loi de 1882 prescrit, en effet, que ce sont sculement les berges des torrents qui seront reboisées les premières. Ce travail de reboisement n'est pas près d'êtıre terminé, car', d'après des calculs faciles ì faire il faudra encore cinquante ans pour arriver à reboiser l'élendue de tous les perimètres, ce qui entrainera une dépense de 70 millions.

En attendant l'achèvement de ce travail gigantesque, il est bon, je crois, de poser notre programme forestier sur des assises plus modestes ; c'est pourquoi je miassocie pleinetnent aux conclusions qui ont été présentées ici par les différents orateurs qui m'ont précédé à la tribunè

Le reboisement est certainement une gêne pour less populations, reconnaissons-le; pour le leur faire accepter, il faut leur offrir une compensation et cette compensation; nous pouvons la trouver dans les améliorations pastorales.

Vous pouvez être persuadés que, pour ma part, convaincu de la légitimité der la causie et de son utilité, je m'emploierai de toutes mes forces à agrandir le domaine des améliorations pastor'ales ct à rendre de plus en plus agréable aux populatins l'administration forestière qui rend par ailleurs tant de services à l'Etat.

\section{Transports d'Énergie Américains à très haute tension}

\section{Transport d'énergie de la Bay Counties Power Co}

Le transport d'énergie à grande distance des Comtés de la baie de San-Francisco, en Californie, a montré, en fonctionnant avec régulatité jusqu'à une distance de 357 kilomètres ( 222 milles), ce qu'on pouvait attendre, dès Igor, d'une transmission de ce genre. Depuis I gor, grâce en partie a cet exemple, les entreprises de transport d'énergie à de très grandes distances se sont multipliées partout où des centres industriels de quelque importance pouvaient être desservis; des applications encore plus nombreuses sont à l'étude, et on peut prévoir que, grâce au bon marché de l'énergie ainsi fournie, une nouvelle ère va s'ouvrir pour l'industrie, au bénéfice des régions où l'on aura le plus vite compris la portée rćelle de l'évolution qui se dessine.

Il s'agissait d'alimenter en force motrice toute une région comprise entre les montagnes et la mer, où le charbon n'arrive qu'à des prix prohibitifs, et d'y faire vivre les industries les plus diverses : moulins, fonderies, ateliers de construction, mines d'or, éclairage électrique dans les villes ou bourgs, installations hydrauliques municipales, traction des tramways, impression des journaux, etc. La Sierra Nevada offrait ses cours d'eau torrentiels, dont le faible débit est racheté par une 